The aim of this study was to assess whether post-tonsillectomy and adenotonsillectomy pain relief prescribing habits changed at the Trust following MHRA restriction of codeine use in children under 18 years of age and introduction of a pre-printed analgesia prescription.

Method Prior to the MHRA statement, post- tonsillectomy and adeno-tonsillectomy patients were discharged from the Surgical Day Unit (SDC) with regular paracetamol and ibuprofen and as required codeine for days 3-7. Morphine was introduced as a replacement for codeine. An unlicensed morphine 100 microgram $/ \mathrm{ml}$ solution was procured for the smaller doses to ensure accurate dosing and avoidance of ethanol. Dosing guidance was issued Sept 2013 and a preprinted prescription was introduced in Nov 2013 with maximum morphine and paracetamol doses for age to compensate for obesity. Morphine oral solution $10 \mathrm{mg} / 5 \mathrm{ml}$ previously subject to controlled drug writing requirements locally was de-regulated at the Trust in Nov 2013.

Tonsillectomy or adeno-tonsillectomy patients were identified using the theatres booking system. Discharge analgesia was recorded from the discharge prescriptions and pharmacy dispensing system. Data collection periods were September to December for 2012 and 2013. Weekend patients were excluded due to potential use of pre-pack analgesia from the ward.

Results In total 374 post adenotonsillectomy or tonsillectomy prescriptions were reviewed, 147 from 2012 and 227 from 2013. In the 2012 group 116/147 (79\%) received codeine, with $107 / 147$ (73\%) having the three analgesics, paracetamol, ibuprofen and codeine on discharge.

In 2013 only 85/227 (37\%) received all three analgesics. Prior to the pre-printed prescription $46 / 128(36 \%)$ received an opioid, with $25 / 128(19.5 \%)$ being given morphine. Codeine was prescribed for 21/128 (16\%) of patients during Sept 2013 regardless of the morphine guidance available in the surgical areas.

Post introduction in of pre-printed prescription morphine issues increased to $56 / 99$ (56.5\%). No correlation was seen for age or weights of patients where morphine prescriptions were not issued in 2013. No codeine prescribed from end of Sept 2013 for patients included in the study.

Conclusions Fewer opioid prescriptions were issued during the immediate change over period in comparison to the same time period in 2012; possibly due to unfamiliarity with drug dosing or the need for controlled drug writing requirements on prescriptions. Introduction of a pre-printed prescription improved the morphine prescribing rates but not to the level of opioid prescribing pre-MHRA codeine restriction era. Re-presentation rates due to inadequate pain relief or other complications are being investigated following the changes in prescribing habits.

\section{P20 IMPACT OF CODEINE RESTRICTION ON POST-OPERATIVE PAIN RELIEF PRESCRIBING}

Julia Dietsch, Rhian Isaac, Dannie Seddon, Ursula Disckson. Birmingham Children's Hospital NHS Foundation Trust

\subsection{6/archdischild-2015-308634.28}

In July 2013 the Medicines and Healthcare Regulatory Agency (MHRA) issued guidance for restricted use of codeine in the paediatric population with contraindication for under 18 year olds with obstructive sleep apnoeas. ${ }^{1}$ Surgical management of OSA includes tonsillectomy where $25-50 \%$ of children experience severe pain. ${ }^{2}$ 